\title{
MEASURING THE MASS OF SMALL ARTHROPOD MUSCLES*
}

\author{
By BRent D. Opell \\ Department of Biology, \\ Virginia Polytechnic Institute and State University, \\ Blacksburg, Virginia 24061
}

There are balances sensitive enough to measure the masses of small arthropod muscles, but preparing and handling these tissues can be problematic. It is often difficult to remove intact muscles and, once removed, they are easily damaged. The method described in this paper can be used to determine the mass of free muscle tissue, muscles that are left attached to pieces of the exoskeleton for easier handling, and muscles within intact arthropod appendages. This method employs a spectrophotometer to measure the amount of dye released when stained muscle tissue is dissolved in sodium hydroxide.

A number of biological stains may prove useful in this technique, although they must be able to withstand an extremely high $\mathrm{pH}$. The example presented below uses Wilkey's doublestain, purchased from Arthropod Slidemounts, P.O. Box 185, Bluffton, IN 46714. This stain contains $5 \mathrm{ml}$ of $5 \%$ aqueous acid fuchsin and $10 \mathrm{ml}$ of $5 \%$ aqueous lignin pink in $100 \mathrm{ml}$ of modified Essig's aphid clearing fluid (Essig 1948), consisting of 20 parts $85 \%$ lactic acid, 2 parts liquified phenol, 4 parts glacial acetic acid, and 1 part distilled water.

To test this procedure, I determined the first leg muscle masses for a developmental series of the spider Miagrammopes pinopus Chickering (Uloboridae) from the U.S. Virgin Islands (Opell 1987). These spiders' live weights ranged from 0.63 to $4.27 \mathrm{mg}$. I killed and preserved them in $75 \%$ ethanol, removed their two first legs at the trochanter-femur joint, and stained these legs for 20 days at room temperature in Wilkey's stain. This long staining time assured that the muscles of even the largest legs were fully saturated with stain. To remove stain trapped within hemolymph channels, I then rinsed legs in five changes of $75 \%$ ethanol for one day each. I next transferred them to $0.7 \mathrm{ml}$ of $5 \%$ (weight/volume) $\mathrm{NaOH}$ and digested them at $70^{\circ} \mathrm{C}$ for 90 minutes.

*Manuscript received by the editor May 14, 1990. 
When these samples cooled, I shook them, allowed particulates to settle out, and then measured their transmittance to $480 \mathrm{~nm}$ light with a Milton Roy Spectronic 20. As Figure 1 illustrates, this is the wavelength of maximum absorbance for Wilkey's stain in a 5\% $\mathrm{NaOH}$ solution. After each sample was measured, its digested leg exoskeletons were removed, rinsed in distilled water, and the procedure described above repeated. This second measurement provided an estimate of the amount of stain taken up by the legs' exoskeletons. I subtracted this from the first value to determine the amount of stain taken up by leg muscles.

To establish a calibration curve (Fig. 2) for muscle mass, I dissected out the leg muscles of another uloborid spider, Octonoba sinensis (Simon). The leg muscles of these alcohol-preserved specimens were pooled, stained as described above, and dried in a vacuum desiccator. I then divided them into portions of increasing mass, as measured on a Cahn Model 4700 Automatic Electrobalance, and digested them in $5 \% \mathrm{NaOH}$. When their masses were plotted against their transmittance to $480 \mathrm{~nm}$ light (Fig. 2), the resulting regression is highly significant $(p<0.0001)$. The following formula, derived from this regression, permits muscle mass to be calculated from transmittance:

$\mathrm{mg}$ Dry Muscle Mass $=0.59471-0.00615$ Transmittance at $480 \mathrm{~nm}$.

To determine if the drying procedure used in establishing the calibration curve affected the properties of the stain, I placed $50 \mu \mathrm{l}$ of Wilkey's stain onto each of 20 Nuclepore $10 \mathrm{~mm}$ prefilters (Type P100), dried them in a vacuum desiccator, and left them overnight at room temperature in $4 \mathrm{ml}$ of $5 \% \mathrm{NaOH}$. Each sample was filtered with a Gelman ACRO LC3A disposable filter to remove suspended filter fibers and its transmittance to $480 \mathrm{~nm}$ light measured. Twenty control samples were prepared by placing $50 \mu \mathrm{l}$ of stain into a vial, immediately adding $5 \% \mathrm{NaOH}$, and letting the preparation set overnight. The mean transmittance of dried and wet stain (6.23 and $6.28 \%$, respectively) did not differ significantly when tested with a T-test $(\mathrm{p}>0.78)$. This shows that drying and rehydrating does not alter the properties of Wilkey's stain and that it is appropriate to use a calibration curve derived from stained, dried muscle to infer the mass of stained, wet muscle.

Figure 3 presents the significant regression $(\mathrm{p}<0.0001)$ of the combined muscle mass of the two first legs of $24 \mathrm{M}$. animotus 
1990]
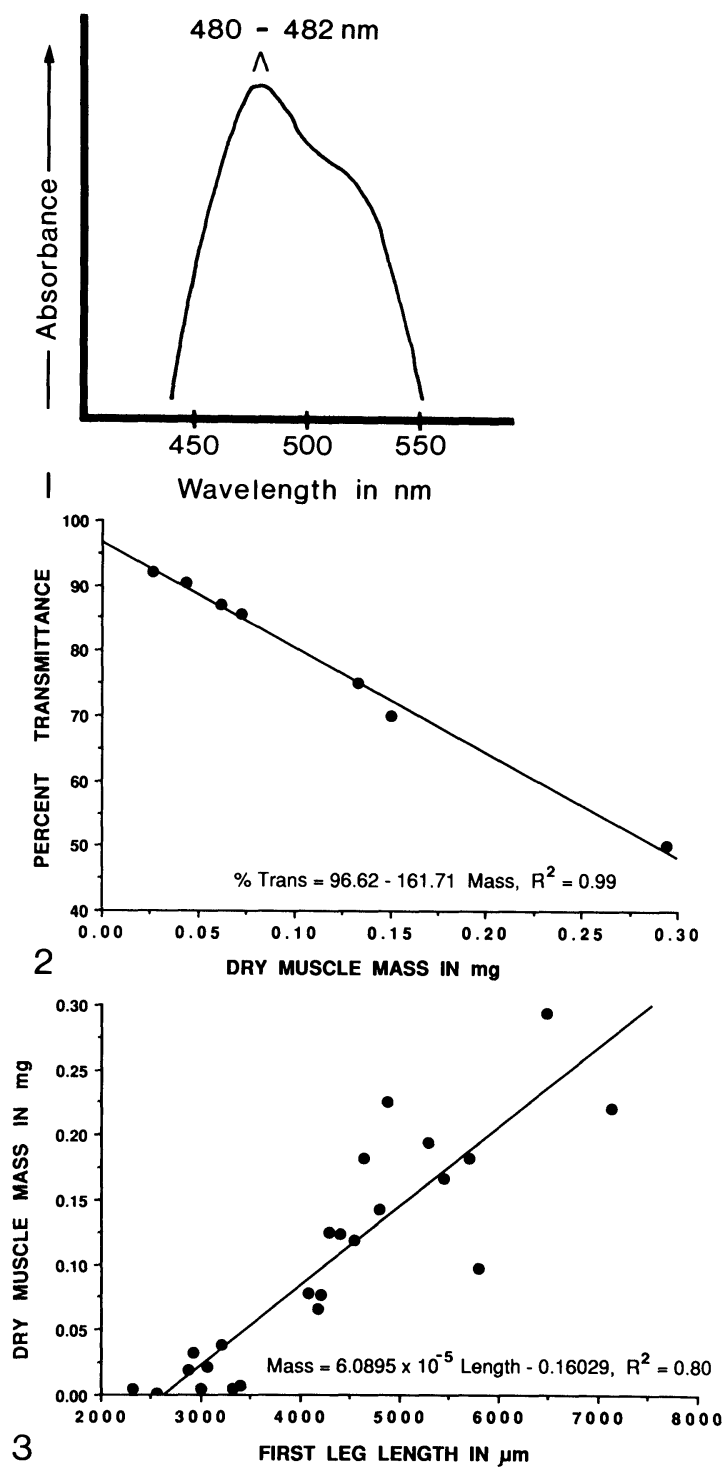

Figures 1-3. 1. Absorbance spectrum of Wilkey's doublestain in $5 \% \mathrm{NaOH}$, as measured with a Beckman Acta MVI Recording Spectrophotometer. 2. Regression of the dry mass of stained Octonoba sinensis leg muscles and their percent transmittance of $480 \mathrm{~nm}$ light after being digested in $\mathrm{NaOH}$. 3. Regression of the combined muscle mass of the two first legs of Miagrammopes pinopus and first leg length. 
against their lengths and shows how the data obtained from this method might be used to characterize developmental changes in leg muscle mass.

Two types of error are inherent in this technique: one tends to slightly overestimate and one to slightly underestimate leg muscle mass. During the initial staining procedure, muscle, hypodermis, and exoskeleton are stained. After $\mathrm{NaOH}$ digestion, only exoskeleton remains to be stained. Because this technique measures the amount of stain taken up by both muscle and hypodermis, it tends to overestimate muscle mass. However, as hypodermis volume is much less than leg muscle volume, this error is small. Additionally, when arthropods of similar size are compared, hypodermis volumes will also be similar.

The second type of error is associated with the alcohol rinses that followed staining. These are intended to be long enough to remove stain from the spaces within even the largest legs, but they almost certainly also leach stain from muscle tissue. Because the same procedure is used both to measure muscle mass and to establish the calibration curve, the amount of leaching is largely controlled for. However, the exoskeleton may retard diffusion of stain from intact leg muscles, causing them to contain more stain than the same mass of free leg muscles. The calibration curve would then slightly underestimate the mass of intact leg muscles.

The technique described above may be more complicated than removing, drying, and weighing muscle tissues, but it is probably at least as accurate. More importantly, it is applicable to small arthropods whose muscle masses could not otherwise be measured.

\section{ACKNOWLEDGMENTS}

Gabrielle Roth and Paula Cushing helped measure and prepare specimens. Partial support for this work came from National Science Foundation grants BSR-8407979 and BSR-8917935.

\section{REFERENCES}

Essig, E. O.

1948. Mounting aphids and other small insects on microscope slides. PanPacific Ent., 24: 9-22.

OPELL, B. D.

1987. Changes in web-monitoring forces associated with web reduction in the spider family Uloboridae. Canadian J. Zool., 65: 1028-1034. 

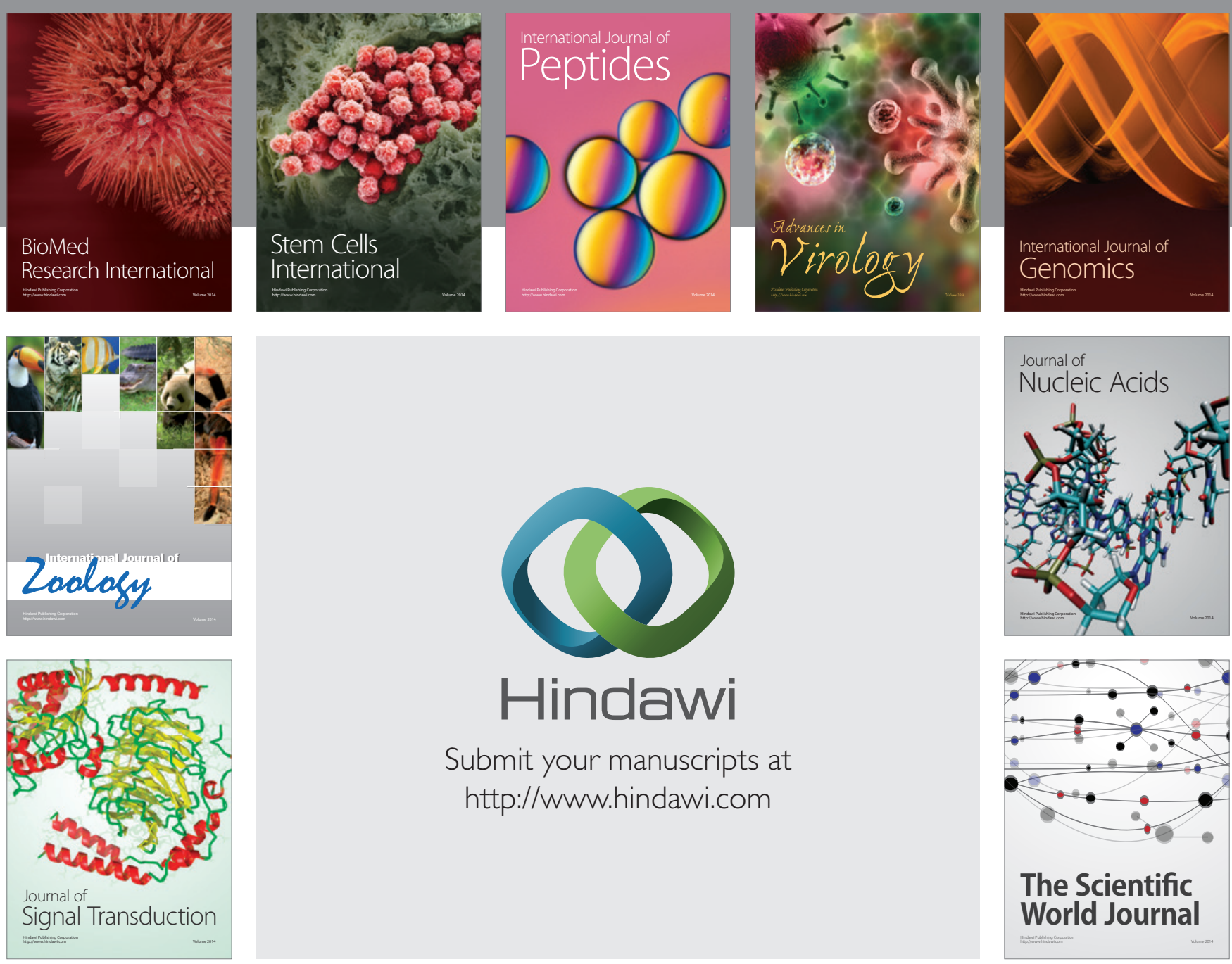

Submit your manuscripts at

http://www.hindawi.com
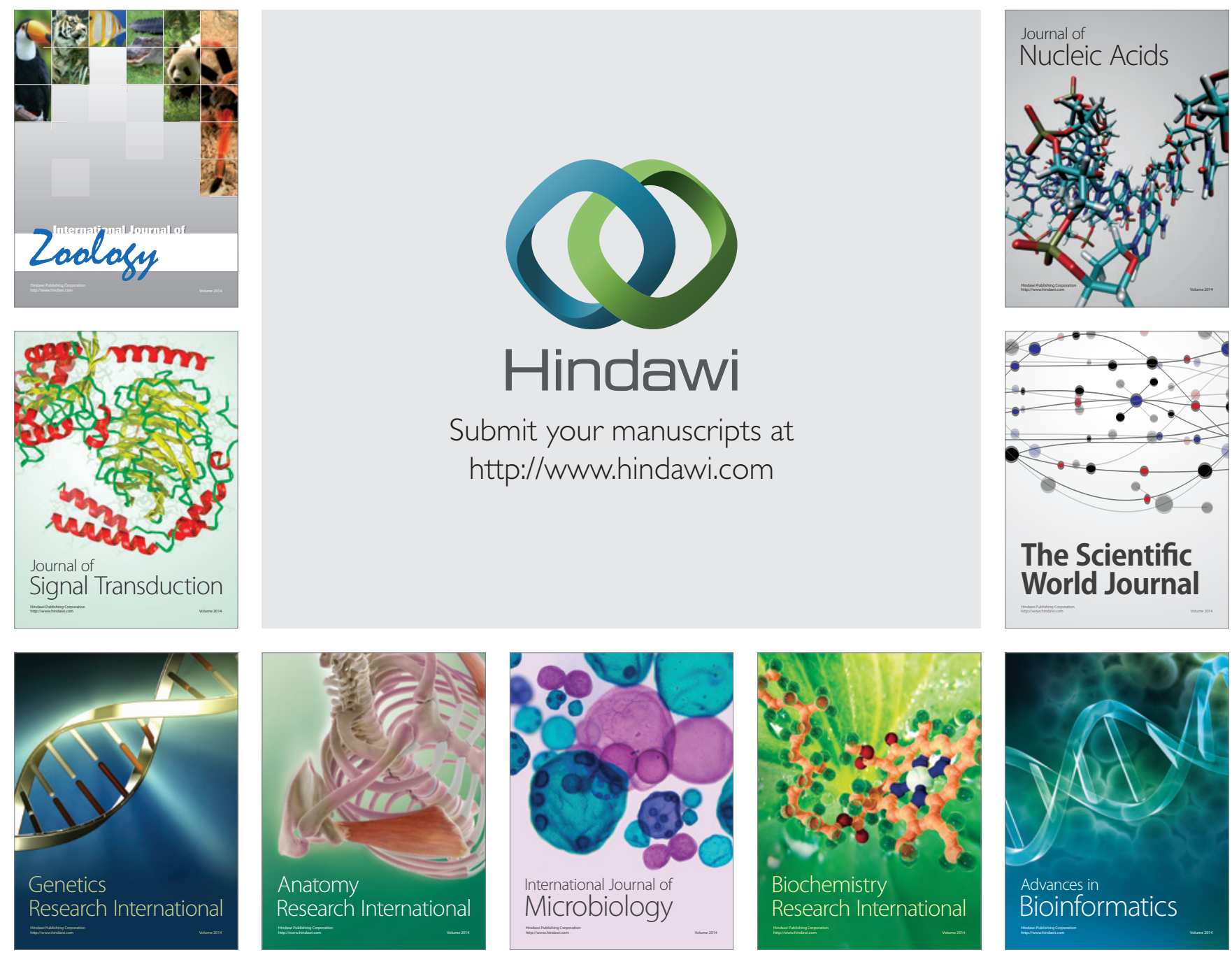

The Scientific World Journal
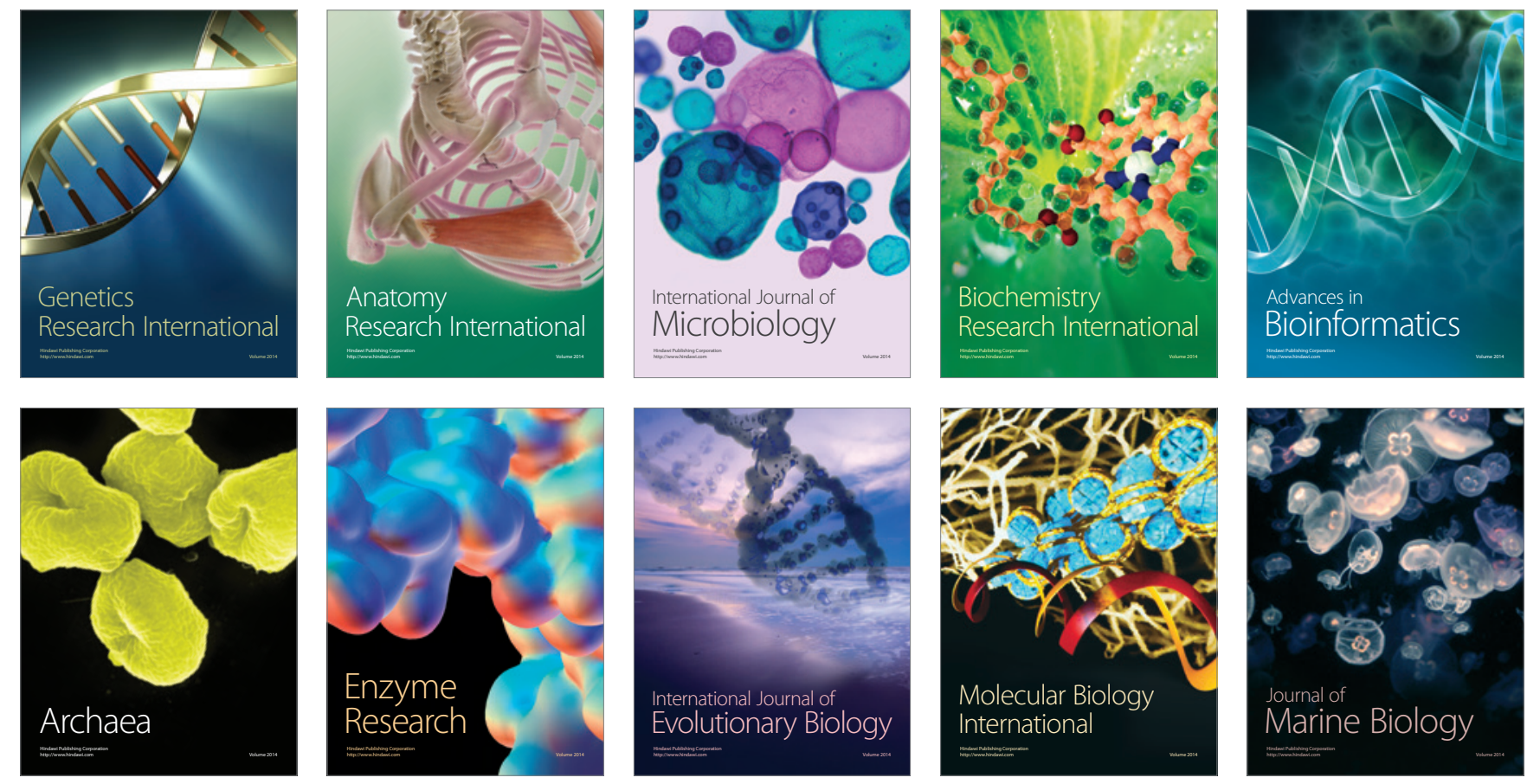